\title{
Bokeh Effect Rendering with Vision Transformers
}

This paper was downloaded from TechRxiv (https://www.techrxiv.org).

\section{LICENSE}

CC BY 4.0

SUBMISSION DATE / POSTED DATE

03-01-2022 / 07-01-2022

CITATION

Nagasubramaniam, Hariharan; Younes, Rabih (2022): Bokeh Effect Rendering with Vision Transformers. TechRxiv. Preprint. https://doi.org/10.36227/techrxiv.17714849.v1

$\mathrm{DOI}$ 


\title{
Bokeh Effect Rendering with Vision Transformers
}

\author{
Hariharan N, Rabih Younes
}

\begin{abstract}
Bokeh effect is growing to be an important feature in photography, essentially to choose an object of interest to be in focus with the rest of the background being blurred. While naturally rendering this effect requires a DSLR with large diameter of aperture, with the current advancements in Deep Learning, this effect can also be produced in mobile cameras. Most of the existing methods use Convolutional Neural Networks while some relying on the depth map to render this effect. In this paper, we propose an end-to-end Vision Transformer model for Bokeh rendering of images from monocular camera. This architecture uses vision transformers as backbone, thus learning from the entire image rather than just the parts from the filters in a CNN. This property of retaining global information coupled with initial training of the model for image restoration before training to render the blur effect for the background, allows our method to produce clearer images and outperform the current state-of-the-art models on the EBB! Data set. The code to our proposed method can be found at: https://github.com/Soester10/ Bokeh-Rendering-with-Vision-Transformers.
\end{abstract}

Index Terms-Bokeh, Transformer, Vision, encoder-decoder, deep learning, EBB!, Resnet.

\section{INTRODUCTION}

Bokeh rendering is an artistic effect in photography to provide the viewers with a perception of foreground and background. This switches the focus of the viewers to the main object providing a shallow depth-of-field while blurring the background and unnecessary details. To produce this effect naturally, the camera's aperture lens is widened and focused on a particular object of choice. However, this requires fast lens with wide aperture, making it almost unattainable for mobile cameras with compact optics and tiny sensors [1]. While high-end smartphones use dual camera set-up for rendering this effect, this still leaves out most of the mobile phones in the market. Thus, to render bokeh images in a small mobile camera, one has to computationally simulate the effect. Existing works, such as PyNet [1] or DDDF [2], utilize additional depth information to simulate realistic bokeh rendering. This, however, poses problem of requiring to compute a depth map of the image in order to simulate the effect. This increases the time and power consumption for the overall process, while also relying on the accurate prediction of depth information of an image from a monocular camera.

In this paper, we propose a method that renders the bokeh effect from a single image without the requirement of a depth map. We use the architecture of recently proposed vision transformers [3] as the backbone to retain the global

Hariharan is with the Department of Computer Science and Engineering, SRM Institute of Science and Technology, Chennai, India (e-mail: hnnhariharan12@gmail.com).

Rabih Younes is with the Department of Electrical and Computer Engineering, Duke University, North Carolina, US (e-mail:rabih.younes@duke.edu).

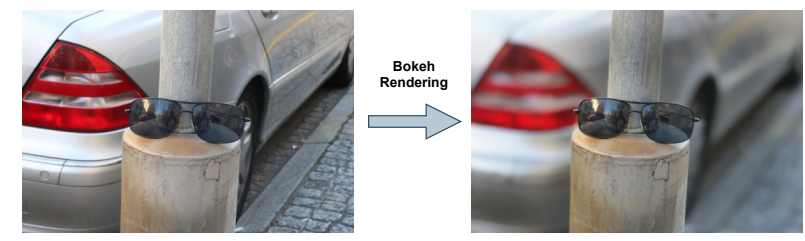

Fig. 1. Visual Sample to demonstrate the Bokeh effect rendered with our method.

information throughout the model layers. For bokeh rendering, it is essential that the model retains maximum features and as close as possible to the native resolution. This mitigates any unrealistic effects on blurring the background and is often helpful in detecting the edges around the foreground. However, with Convolutional Neural Network (CNN) architecture, feature resolution and granularity are lost in the deeper stages of the model and can thus be hard to recover in the decoder [4]. While a CNN uses filters to extract only part of the image and progressively down samples at various stages, a vision transformer works with the entire image, taking patches of these images as input. These patches are used to train the model at different resolutions. But, unlike fully-convolutional networks, the vision transformer backbone foregoes explicit down sampling operations after an initial image embedding has been computed and maintains a representation with constant dimensionality throughout all processing stages [4]. This eventually mitigates the loss of feature granularity that CNNs suffer from.

In this paper, we propose an encoder-decoder architecture with a transformer as the basic building block of the encoder. We leverage the model proposed in [4] to produce image-like feature representations at various resolutions and progressively combine the feature representations into the final prediction using a convolutional decoder. This model also has a global receptive field at every stage, thus allowing for globally coherent predictions. We modify the hooks used for training to assemble features from both early layers and deeper layers of the encoder model. In this way, low level encoders can provide additional global intermediate features, and higher level encoders can provide more local intermediate features to improve the fidelity of generated bokeh images [5]. Additionally, We propose an input to input mapping method during training that helps retain details in the foreground and further enhances the uniformity of blur effect in the background of the bokeh predictions.

Our work achieves state-of-the-art results in Bokeh Rendering on monocular images, when trained on tested on the EBB! dataset [6]. Moreover, our model significantly reduces the computational cost and time by dismissing the use of any 
Depth Estimation or Saliency Detection model. We have also compared the run time of our model with that of the current state-of-the-art methods.

\section{RELATED WORK}

A fully Convolutional Neural Network has always been the backbone of any architecture for bokeh rendering. Many works leverage the depth information obtained from stereo or monocular cameras to render bokeh effect. In the work of [7], the authors obtain a depth map with stereo matching and then apply the bokeh effect. The work of [8] produces a high resolution disparity map and combines it with a rendering algorithm to render bokeh effect. While these works result in a visually pleasing effect, they are not applicable for monocular camera setups.

The work in [9] introduces Monocular Bokeh Effect Rendering using a CNN to obtain segmentation map and then generate depth-of-field image with blurred background on portrait images. Wadhwa et al.[10] proposed a system to computationally synthesize shallow depth-of-field images using person segmentation and a depth from dual-pixel auto focus hardware. This, however, fails to produce photo realistic results due to the use of an approximated disk blur kernel to blur the background. The work of [2] also proposes a monocular bokeh effect rendering method guided by pre-trained salient-region segmentation and depth-estimation modules. They proposed a model composing of an efficient densely connected encoder-decoder with a pyramid pooling module, leveraging the task-specific efficacy of joint intensity estimation and dynamic filter synthesis for the spatially-aware blurring process. The work of [11] uses Gaussian blur kernels and depth map computed using MegaDepth [12] and producing the resultant bokeh image as a weighted sum of the input image and its different blurred versions.

Zheng et al. [13] used a multi-scale predictive filter CNN with Gate Fusion Block, Constrained Predictive Filter Block, and Image Reconstruction Block. With pixel coordinate maps concatenated to the fullscale images, they trained the model with image patches. Xiong et al. [13] used modified U-Net with residual attention mechanism, multiple Atrous Spatial Pyramid Pooling blocks, and Fusion Modules. Yang et al. [13] used two stacked bokehNet with additional memory blocks to capture global and local features. The generated feature maps from each of their models are concatenated and fed to a Selective Kernel Network [14]. These methods, however, cost high computational time.

In the paper [1], the authors introduce a multi-scale end-toend deep learning architecture, PyNet, for natural bokeh image rendering by utilizing both input image captured with narrow aperture and pre-computed depth map of the same image. While this work renders a realistic bokeh effect, this however relies on the information from a depth map. This drastically increases the time required to render the bokeh effect. The work in [5] proposed a fast and efficient network that leverages the encoder-decoder architecture. This end-to-end model renders bokeh effect directly from the monocular image without requiring any Depth information. However, due to the CNN backbone, the model's generated results are not very photo realistic. Convolutions, by definition, are linear operators that have a limited receptive field. The limited receptive field and the limited expressivity of an individual convolution hinders the model to learn from the entire image [4]. This, however, results in loosing most of the image features. In this paper, we propose a model with Vision Transformer backbone with global receptive field at every stage of the encoder block. This ensure the model to retain global information resulting in much sharper and coherent blur effect rendering.

\section{Proposed Method}

In this paper, we propose Vision Transformer as the backbone for the encoder-decoder architecture to learn from the image at whole and present an end-to-end solution for bokeh rendering. We leverage the DPT model proposed in [4] to construct the transformer encoder, reassemble, and fusion layers for our architecture. The overview of this model is shown in Figure 2.

Transformer models take a strand of strings or tokens as input. The Vision Transformer backbone [3] also works in a similar way by taking in patches of the image as input. These patches are then flattened into vectors and linearly projected to be fed into the encoder. These image embeddings are then concatenated with a positional embedding to allow the model to learn the position of each of these patches in the final image. We follow this approach for a feature map output from ResNet50 feature extractor [15]. The transformer model also has a global receptive field at every stage after the initial embedding. While CNNs progressively increase their receptive field as features pass through consecutive convolution and downsampling layers, our model maintains the spatial resolution of the initial embedding throughout all transformer stages [16], [4]. We divide the input images into patches with a size of $16 \times 16$ and flatten it to a dimension of 768. Since this dimension is greater that the number of pixels in the input patch, it allows the embedding procedure to retain more information. This allows the features from the input patches to be resolved with pixel-level accuracy [4]. These embeddings are then feed it into an encoder containing 12 transformer layers. Each of these layers features its own Multi-Head Attention layer [16]. At the initial stages of training, we use hooks to extract the output of 4 of these layers, specially 2 from the top layers to retain global intermediate features at higher resolution and 2 from deeper layers for local intermediate features at lower resolution, similar to the DPT model [4]. Upon stage 1 of training, we modify the hooks such that the encoder outputs are obtained solely from the early stages to fine tune the model at higher resolution. This allows the model to better learn the edges of the foreground for providing a realistic bokeh effect.

The Decoder consists of Reassemble and Fusion layers from the work of [4]. The output from the encoder blocks 


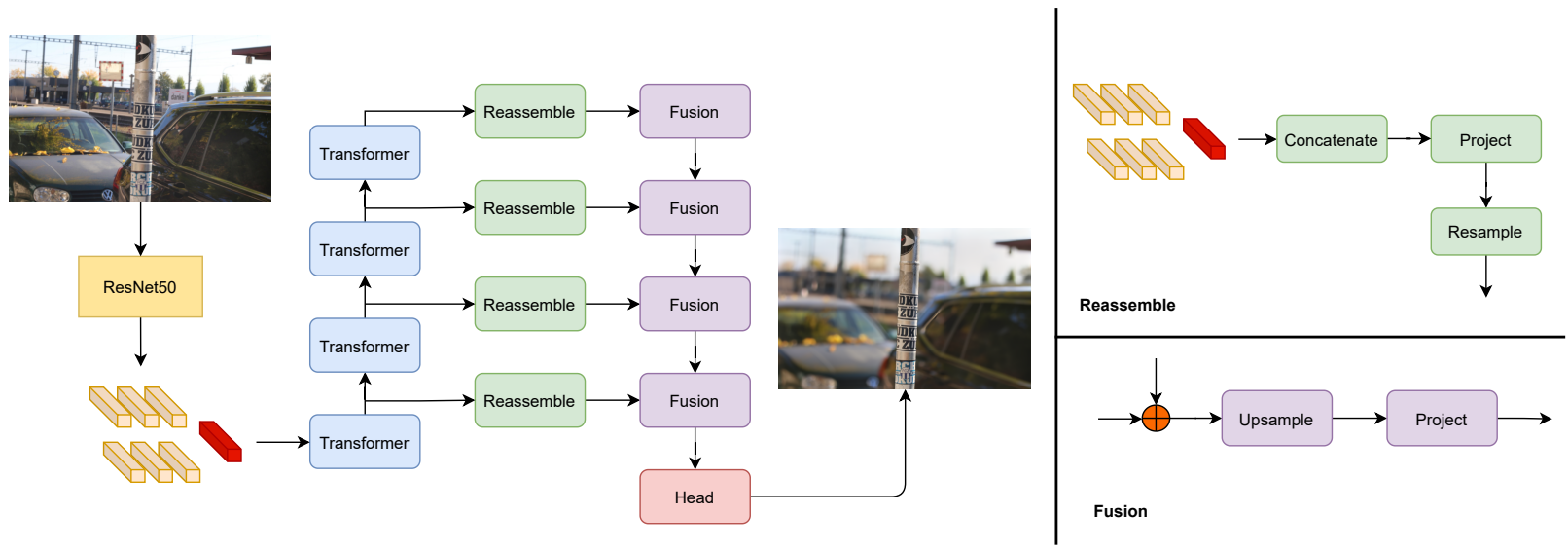

Fig. 2. Left: Architecture Diagram for our method adapted from [4]. The input image is directly fed into a ResNet-50 feature extractor. These feature maps are then divided into non-overlapping patches (orange) and converted into tokens. Positional Embeddings and Readout Tokens(red patch) are then added before passed on to the transformer blocks (blue). Output tokens are reassembled from 4 different stages into image-like representation at multiple resolutions with the Reassemble blocks(green). Fusion layers(purple) are used to fuse and progressively up sample the result. The Head layer(red) coverts the prediction into an RGB Image. Right: Overview of Reassemble and Fusion Blocks.

are assembled at various resolutions. This forms an image like representation that is progressively fused into the final prediction. This final image is the output bokeh effect rendered for the input image at the same resolution. We use the readout project variant for the readout token to capture and distribute global information in the decoder. We then follow the re-sample and concatenation methods proposed in [4] for the reassemble layers. This reassembling of features is done at four stages with each at different resolution. This is achieved by specifying the hooks to determine the output of the transformers which will be passed on to the decoder model. This layer produces a feature map with 256 dimensions, which are then progressively combined and upsampled in each stage of the fusion block. A final convolutional layer is then added to the head to render the final bokeh prediction at the same resolution of that of the input image.

\section{EXPERIMENTS}

In this paper, we introduce a new method of training a model to render any effects while maintaining the specific details from the input image. We initially train the model to map back to its input. The model is trained to retain all its input features before being mapped to the actual output. This significantly improves the fidelity of the predicted output and provides sharper and clearer images. With input-to-input mapping, the model tends to output a natural looking blurred effect. Due to being an end-to-end model, this method also retains any small details in the input image, that may be lost due to the bokeh effect. Since it is easier to train a model to learn to predict the same input, this method is also much faster compared to directly training the model with the corresponding inputs and outputs. Since the bokeh rendering problem relies on the model to retain most features of the input image, this training method produces state-of-the-art results with the proposed Vision Transformer model.

\section{A. Dataset}

Our model is trained on the Everything is Better with Bokeh (EBB) [6] dataset. EBB is a large-scale bokeh dataset consisting of 5094 pairs of original and their corresponding bokeh images with an average resolution of $1024 \times 1536$. For each pair, the original image was captured with a narrow aperture (f/16) that results in a normal sharp photo, whereas the bokeh image was shot using the highest aperture (f/1.8) leading to a strong blur effect. The photos were taken during the daytime in a wide variety of places and in various illumination and weather conditions. Since the test ground truth images are not yet available for public, we use the Val294 set [11] for testing and evaluations. All the compared models have also been tested on the Val294 for clear contrast.

\section{B. Training and Testing}

Our model has been implemented using Python and Pytorch [17] and have been trained and tested on the NVIDIA RTX 3080 GPU with 10GB of VRAM. We split 4694 pairs of training data available from EBB as 4400 for training and Val294 set for validation. Furthermore, to leverage the potential of a Transformer backbone, we augment and increase the training set by applying horizontal and vertical flips. The entire training set is rescaled to $768 \times 512$ pixels and trained with a batch size of 1 .

We break our training process into two stages. The first stage consist of the input-to-input mapping. We train the model for 20 epochs until the loss value stabilizes. For the second stage, we change the output to the bokeh images and train the for 40 epochs with lower learning rate. This method of training helped the model to refrain from loosing essential information in the process of applying the bokeh effect. This also helped the model to formulate the result images faster and required fewer epochs to train. 

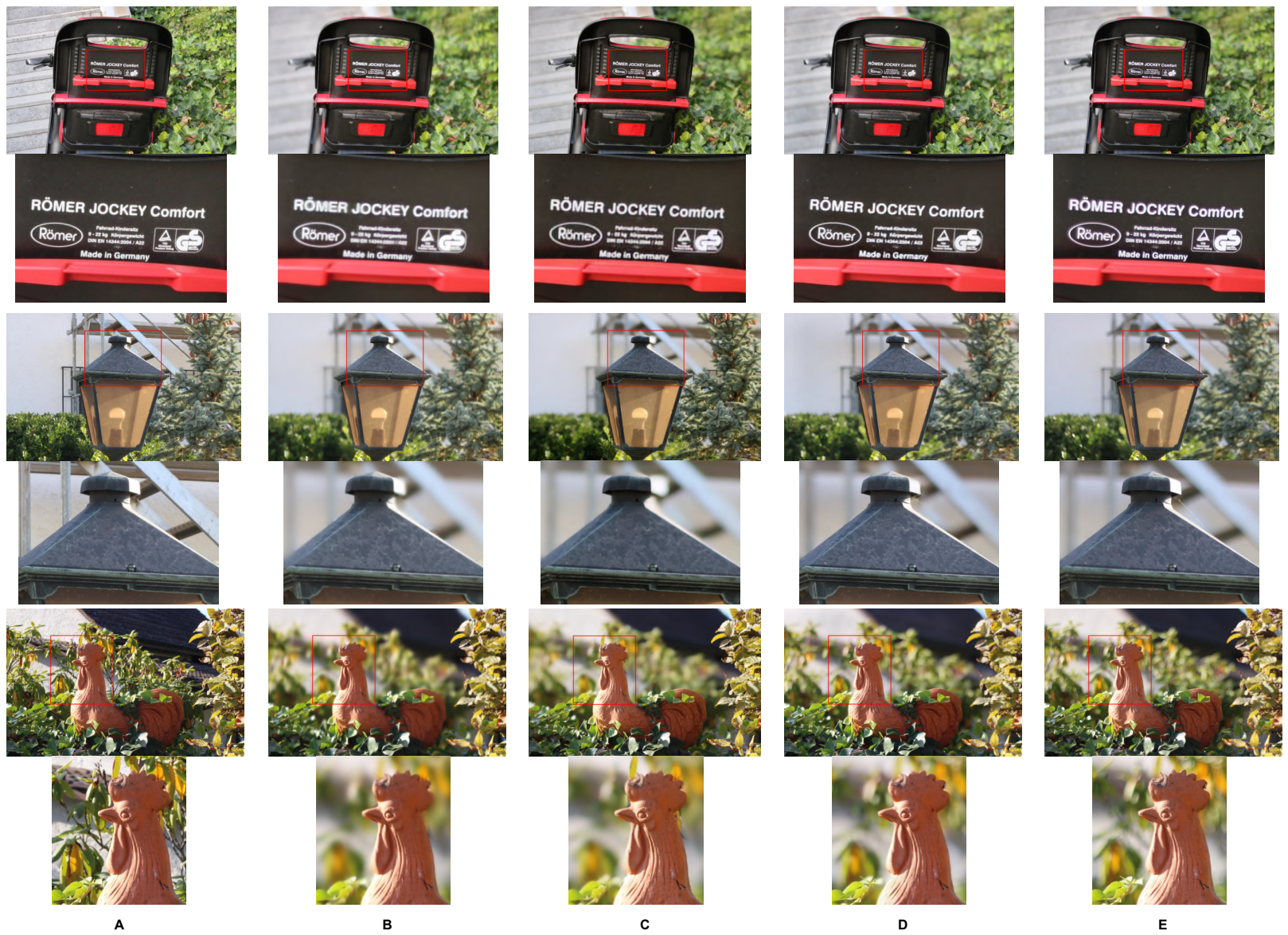

Fig. 3. Qualitative Comparison of Image Restoration and Foreground detail. A: Input Image, B: DMSHN, C: PyNet, D: Ours, E: Ground Truth.

\section{Loss Functions}

In this work, we utilize 3 loss functions and implement them in four steps. For the first stage of training with input-to-input mapping, we train the model with the L1 loss function [18]. During the second stage, we train the first 10 epochs with the L1 loss function, the next 20 epochs with linear combination of L1 and SSIM loss functions [18], as proposed in [5], and the final 10 epochs with MS-SSIM loss function [19].

\section{Metrics}

We aim not only to reduce the computational time, but also to produce visually pleasing results. To demonstrate this, we compared the performance of our model to that of the current state-of-the-art methods such as PyNet [1], DMSHN [5], DBSI [11], and SKN [14]. The following metrics were used to compare the methods:

1. Peak signal-to-noise ratio (PSNR) [20]: ratio of the maximum value of the pixel to the noise that affects the quality of the pixels. The higher the value of PSNR, the better the quality of the output image.

2. Structural Similarity (SSIM) [18]: to quantify the similarity in quality of two images. The higher the value of SSIM, the better the quality of the output image.

3. Learned Perceptual Image Patch Similarity metrics (LPIPS) [21]: Distance evaluation between image patches. The lower the value of LPIPS, the similar the image patches.

4. Mean Opinion Scores (MOS): Following the work of [1], [5], we also evaluate our work based on a study conducted with 20 users. The users were presented with 20 randomly selected bokeh images and the predictions from each model. The users were then asked to score the predictions on a scale of 0 to 4, 0 being "identical" and 4 being "different", in comparison with the corresponding bokeh images.

5. Mean Opinion Ranks (MOR): To further evaluate the visual quality of the models, we present the user with the predictions of 10 random images from the EBB test set. Due to the lack of ground truth for these images, the users were asked to compare and contrast the results from PyNet [1], DMSHN [5], and our model especially with the focus on foreground, edges between foreground and background, blur effect, and the quality and clarity of the predicted images. The users were then asked to rank the results as 1,2 , and 3 . The average ranking of all the images by all the users are computed for each model. The lower the average ranking, the better the model's results.

\section{E. Results}

We test all the models against the Val294 set and Table I shows that our model outperforms the current state-of-the-art methods. Furthermore, it can be seen that our method has also scored 1.19 in MOS and 1.60 in MOR to demonstrate a better image restoration with sharp detail in the foreground and a 


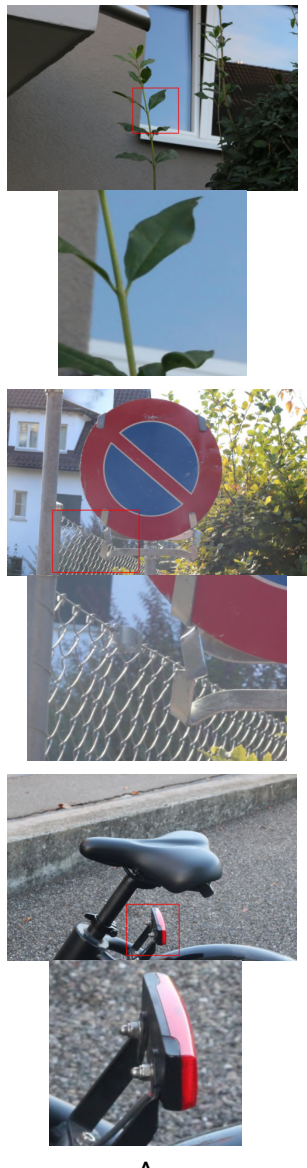

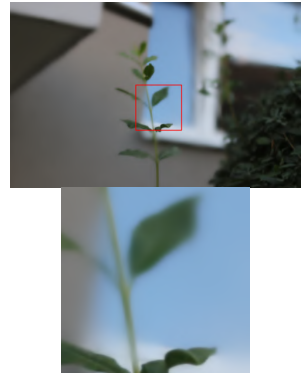
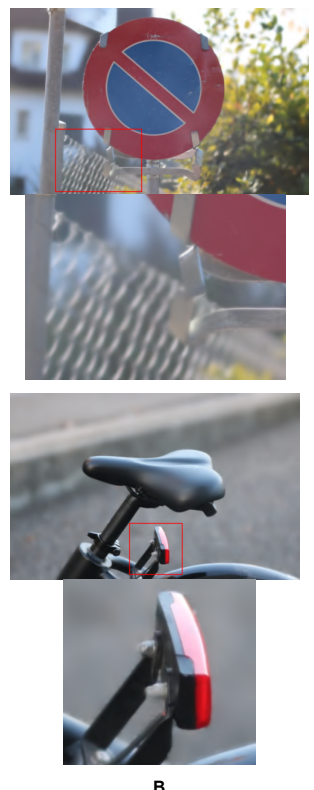
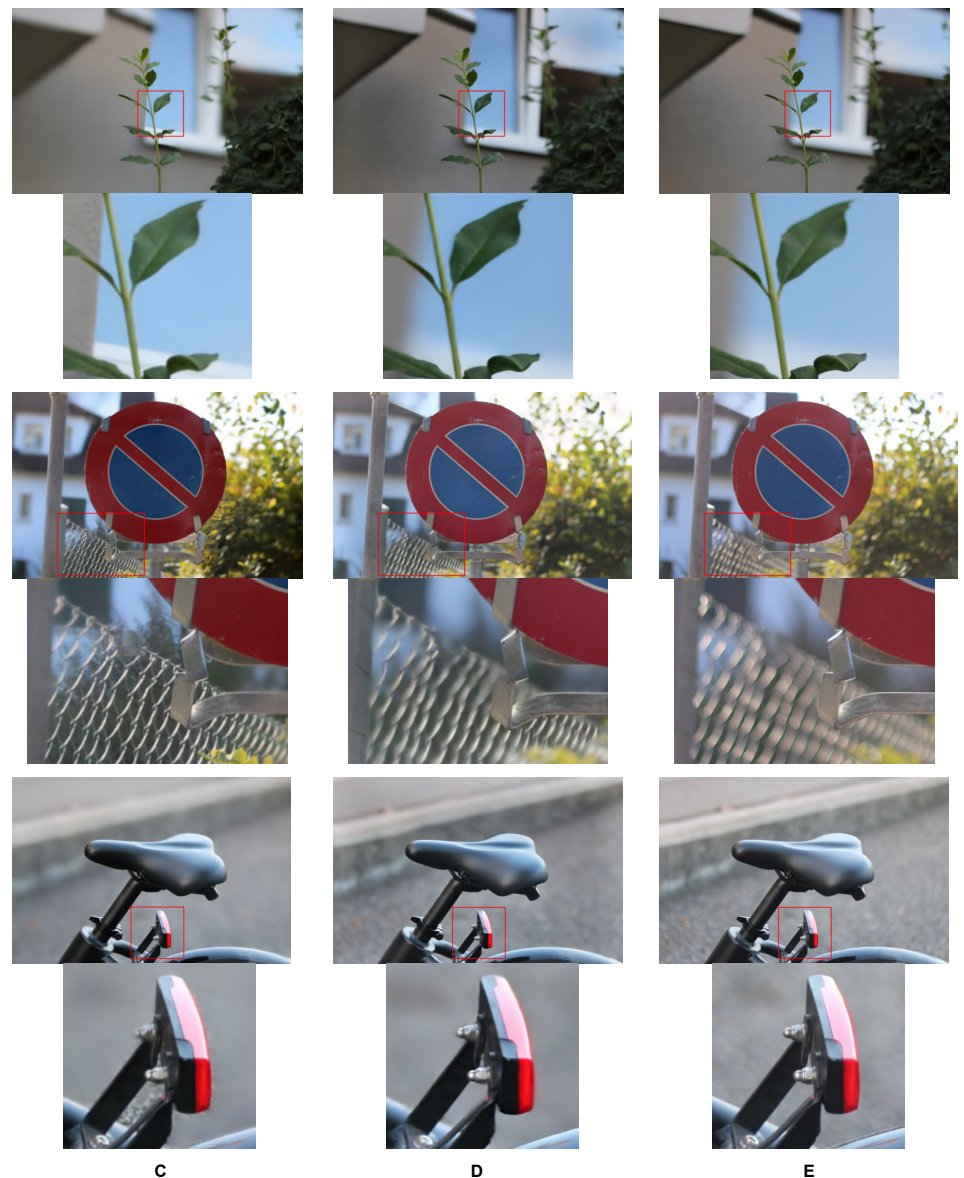

Fig. 4. Qualitative Comparison of Bokeh Effect along the edges of the foreground and in small gaps between the foreground and the background. A: Input Image, B: DMSHN, C: PyNet, D: Ours, E: Ground Truth.

TABLE I

Metrics Result Comparison With EBB VAL294 SET. THE Best RESULTS ARE REPRESENTED IN BOLD AND THE SECOND BEST ARE UNDERLINED.

\begin{tabular}{|c|c|c|c|c|c|}
\hline Method & PSNR & SSIM & LPIPS & MOS & MOR \\
\hline SKN [14] & 24.66 & 0.8521 & 0.3323 & - & - \\
\hline DBSI [11] & 23.45 & 0.8675 & 0.24633 & - & - \\
\hline DMSHN [5] & 24.72 & $\underline{0.8793}$ & 0.2271 & 1.62 & 2.73 \\
\hline PyNet [1] & $\mathbf{2 4 . 9 3}$ & 0.8788 & $\underline{0.2219}$ & $\underline{1.38}$ & $\underline{1.67}$ \\
\hline \hline Ours & $\underline{\mathbf{2 4 . 7 6}}$ & $\mathbf{0 . 8 9 0 4}$ & $\mathbf{0 . 1 9 2 4}$ & $\mathbf{1 . 1 9}$ & $\mathbf{1 . 6 0}$ \\
\hline
\end{tabular}

uniform bokeh effect for the background. We demonstrate this in Figure 3 and by comparing the output images of the models. Our proposed method show a significant improvement in the visual quality as well as the metrics when compared to the current state-of-the art methods. It also retains the small details and information better, especially with text. For the tests conducted, it was also noted that there was very minimal blur effect leak in the foreground, when compared to the other models. Our model was able to identify the foreground from the background even in small gaps as shown in Figure 4 with many images.

We also test the models on unseen EBB test data set and compare the qualitative performance. Since the ground
TABLE II

PARAMETERS AND RUNTIME COMPARISON WHEN TEST ON RTX 3080 GPU. THE BEST RUNTIME RESULT IS REPRESENTED IN BOLD AND THE SECOND BEST IS UNDERLINED.

\begin{tabular}{|c|c|c|}
\hline Method & Parameters (M) & Runtime (s) \\
\hline DMSHN [5] & 10.84 & $\mathbf{0 . 0 3 3 4}$ \\
\hline PyNet [1] & 47.5 & 0.2518 \\
\hline \hline Ours & 123.14 & $\underline{0.0369}$ \\
\hline
\end{tabular}

truth for this set was unavailable, we ask the users to rank the output images from different models. Figure 5 shows few samples and their corresponding predictions from each model. It demonstrates the ability of our model to identify the foreground on unseen images better when compared to the current state-of-the-art methods such as PyNet [1] and DMSHN [5]. Our work better retains the details while minimizing the blur effect leaking on to the foreground. It also shows a consistent blur effect in the background without any sharp contrasts or hard blurring, refraining from looking artificial.

Our model consists of 123.14 million parameters and takes about 0.3 to 0.4 seconds to predict the output of a single image when tested on NVIDIA RTX 3080 GPU with 10GB 

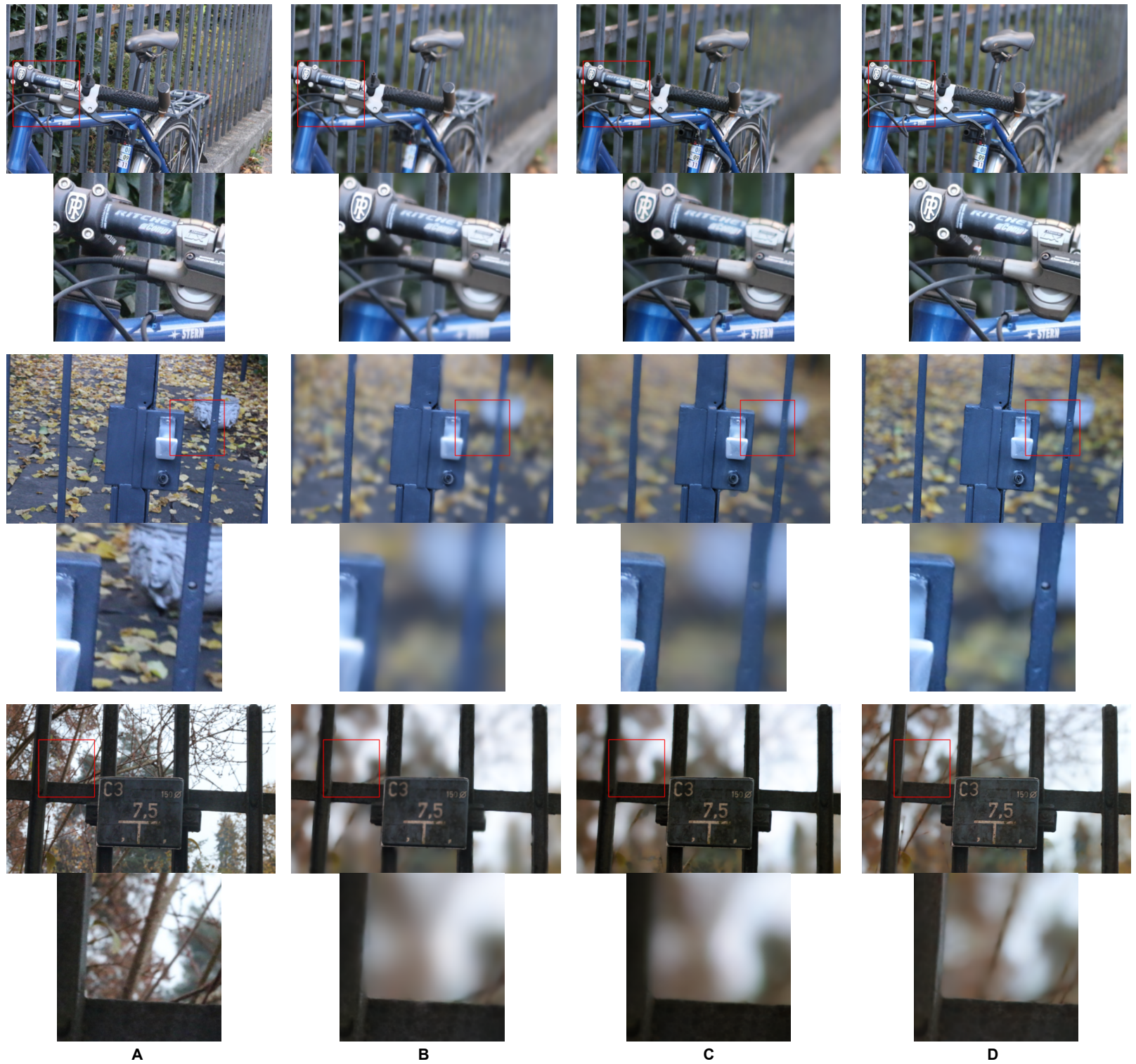

Fig. 5. Qualitative Comparison of Bokeh Rendering with random test data from the EBB Test set. Since ground truth was not available, MOR metric was used on this set to determine the model that produced the most photo-realistic results. A: Input Image, B: DMSHN, C: PyNet, D: Ours.

of VRAM. Table II shows the runtime and parameters comparison when tested on the same GPU.

\section{F. Ablation Study}

To justify our technical choices, we show the various ablation study conducted during the implementation of our model.

Image Restoration Training: To train the model to better retain the details of the foreground, we initially train the model to predict the same input that was fed into the model. This allow the model to familiarise with the input images and are then introduced to train for rendering the bokeh effect. This not only allows the model to retain the details better in the foreground, but also significantly reduces the training time and the number of epochs required to achieve the desired
TABLE III

METRICS RESULT COMPARISON WITH AND WITHOUT INITIAL INPUT-TO-INPUT MAPPING ON EBB VAL294 SET. THE BEST RESULTS ARE REPRESENTED IN BOLD.

\begin{tabular}{|c|c|c|c|}
\hline Method & PSNR & SSIM & LPIPS \\
\hline Ours (without Input-to-Input Mapping) & 22.88 & 0.8516 & 0.2558 \\
\hline Ours (with Input-to-Input Mapping) & $\mathbf{2 4 . 7 6}$ & $\mathbf{0 . 8 9 0 4}$ & $\mathbf{0 . 1 9 2 4}$ \\
\hline
\end{tabular}

results. The model learns to predict the results much quicker than when trained directly on the bokeh images. Table III shows the results of our models when trained with and without the initial input-to-input mapping. Both models were trained for 50 epochs, while the first 20 epochs of Model 2 was trained with input-to-input mapping. The difference in predictions from the models can be seen in Figure 6, demonstrating better foreground detail retention in the model 

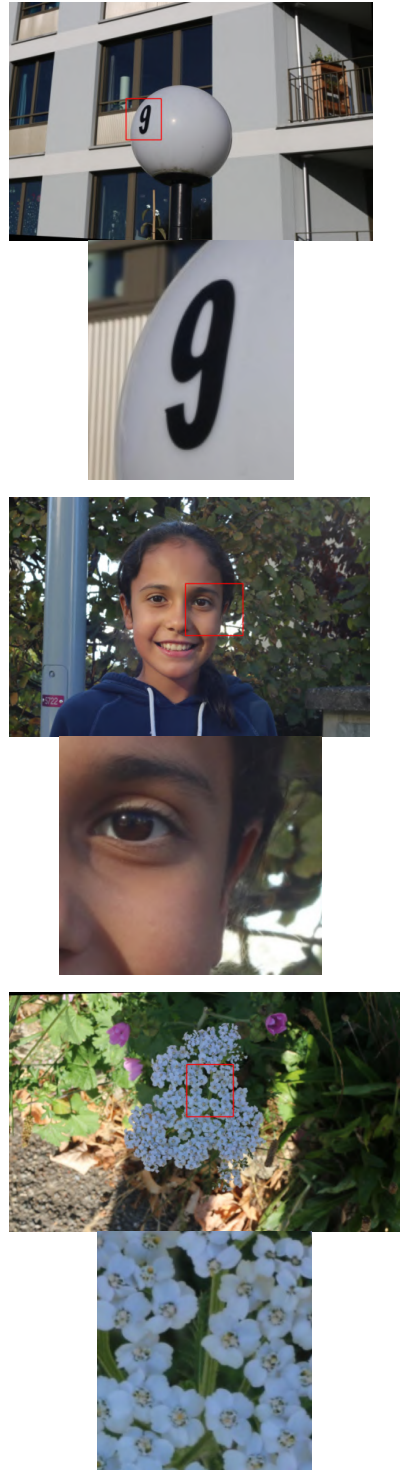
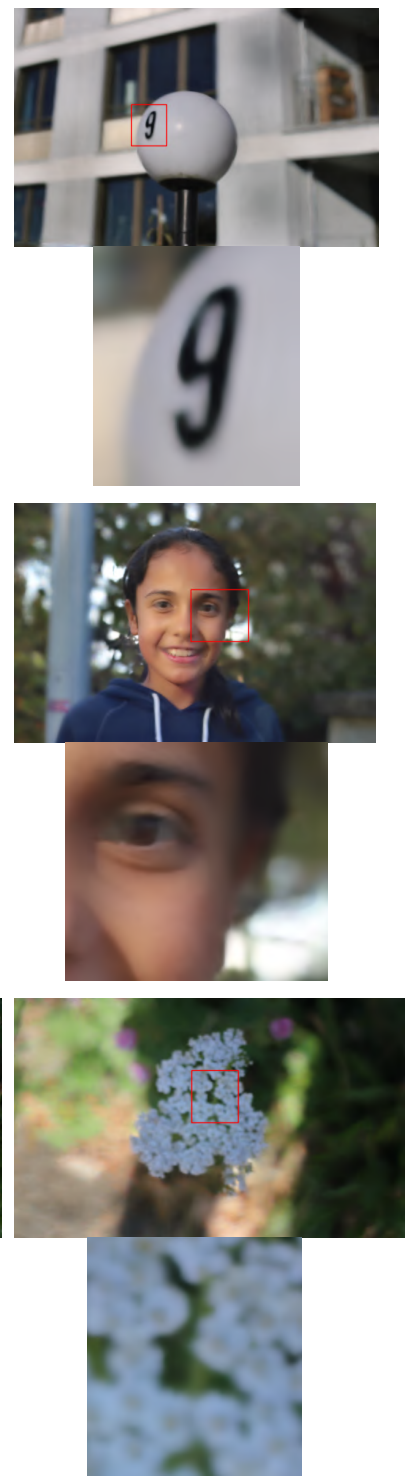

B
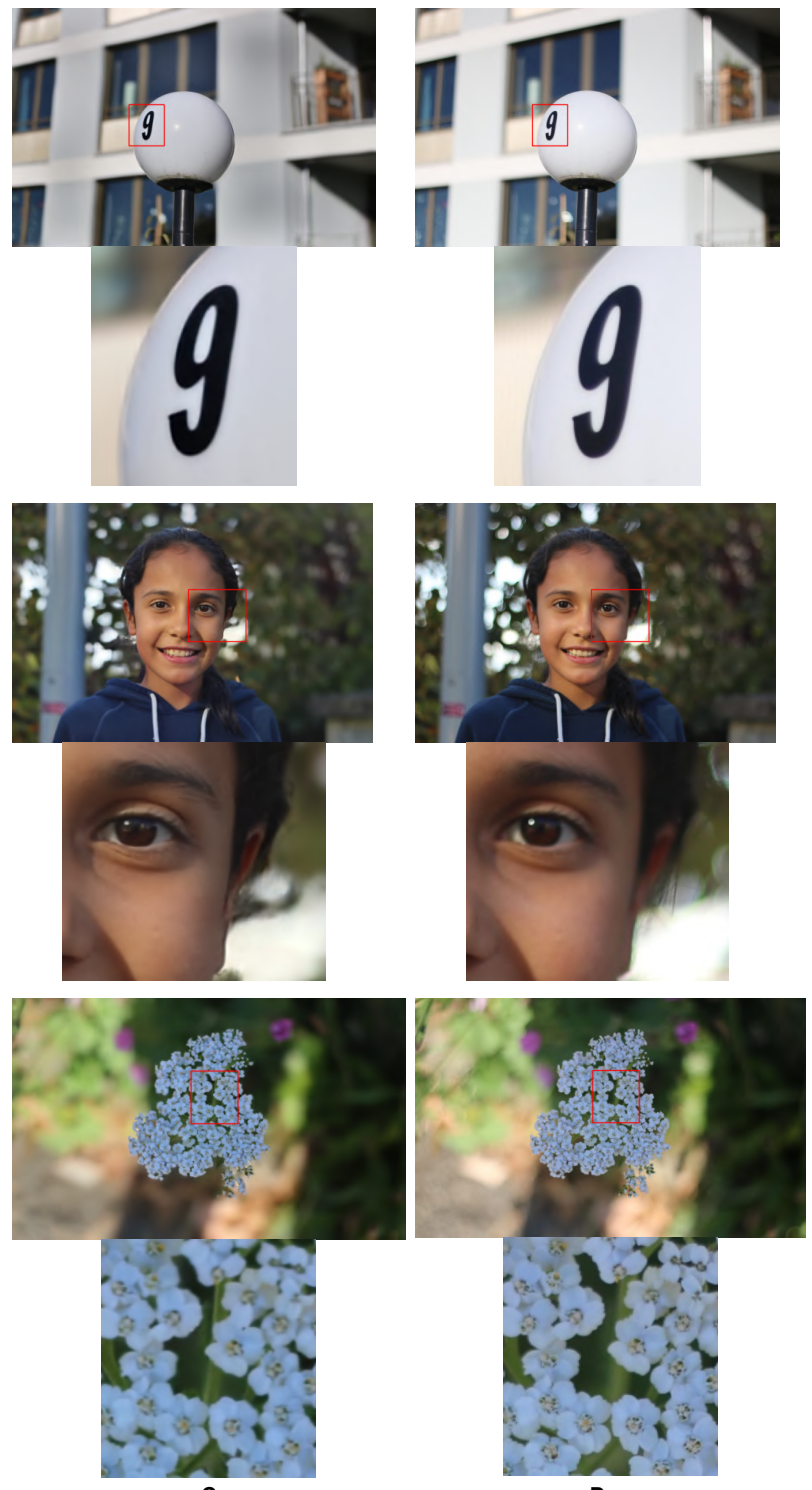

D

Fig. 6. Qualitative Comparison of Bokeh Effect with and without initial input-to-input mapping. A: Input Image, B: Ours (Without Mapping), C: Ours (With Mapping), D: Ground Truth.

when trained with input-to-input mapping. It can also be noticed that the blur leak is minimized when trained initially with input-to-input mapping and the bokeh effect for the background seem more uniform and natural.

Skip Connections: Adapting from the work of [4], we also utilize the tokens from a mixture of layers containing low-level features as well as high level features. We use hooks to obtain the tokens from the top layers and the deeper layers of the encoder. This allows the model to learn from both high resolution and low resolution images. However, upon learning for from this method for the first 40 epochs, we switch the hooks to allow the model to learn from high resolution alone. For the last 10 epochs of the training process, we obtain only high level features from the top layers of the encoder. This showed a improved performance after the initial stage, allowing the model to continue learning before stabilizing.
Learning Rate: We use the Adam optimizer [22] with two sets of learning rate. We initially train the model with a learning rate of $1 \mathrm{e}-5$ for 40 epochs. For the last 10 epochs, we reduce the learning rate to $1 \mathrm{e}-6$ to allow the model to continue learning slowly and further improve the learning curve.

Loss: The first 30 epochs are trained with L1 loss function. Once the rate of learning for the model starts to stabilize, we change the loss function to a linear combination of L1 loss function and SSIM loss function [5] for the next 10 epochs. After this stage, the model starts to stabilize, showing little to no improvements. We then change both the loss function and the learning rate of the optimizer to allow the model to show small improvements. The next 10 epochs were trained with the MS-SSIM loss function, until the the learning curve 
of the model stabilizes.

\section{CONCLUSION AND Future WORK}

In this paper, we introduce an end-to-end solution with Vision Transformers for Bokeh rendering. Dismissing the use of Depth maps, our method presents a fast yet realistic bokeh effect for applicable images. Furthermore, we present a new input-to-input mapping method for image restoration problems to increase the chance of retaining details from the original image, while also reducing the number of epochs required to achieve the desired results. We establish new state-of-the-art results with this method. In addition, we also show that our model's results are clearer and sharper than that of the other models. It is also faster than the models that rely on depth maps, such as PyNet [1], and is comparable to the speed of DMSHN [5].

In future, the model can be improved by training with a larger dataset as Transformers learn better when trained on large datasets. We can also explore the model's performance when trained on high resolution images. The model can furthermore be modified to incorporate the depth information from the image in order to provide more visually pleasing results at the cost of memory and time. Image segmentation can also be incorporated to better understand the subject and detect the edges that differentiate the foreground from the background. This could potentially help preserve the details in the subject while rendering a uniform bokeh effect for the background.

\section{REFERENCES}

[1] A. Ignatov, J. Patel, and R. Timofte, "Rendering natural camera bokeh effect with deep learning," in Proceedings of the IEEE/CVF Conference on Computer Vision and Pattern Recognition Workshops, 2020, pp. 418 419.

[2] K. Purohit, M. Suin, P. Kandula, and R. Ambasamudram, "Depth-guided dense dynamic filtering network for bokeh effect rendering," in 2019 IEEE/CVF International Conference on Computer Vision Workshop (ICCVW). IEEE, 2019, pp. 3417-3426.

[3] A. Dosovitskiy, L. Beyer, A. Kolesnikov, D. Weissenborn, X. Zhai, T. Unterthiner, M. Dehghani, M. Minderer, G. Heigold, S. Gelly et al., "An image is worth $16 \times 16$ words: Transformers for image recognition at scale," arXiv preprint arXiv:2010.11929, 2020.

[4] R. Ranftl, A. Bochkovskiy, and V. Koltun, "Vision transformers for dense prediction," arXiv preprint arXiv:2103.13413, 2021.

[5] S. Dutta, S. D. Das, N. A. Shah, and A. K. Tiwari, "Stacked deep multi-scale hierarchical network for fast bokeh effect rendering from a single image," in Proceedings of the IEEE/CVF Conference on Computer Vision and Pattern Recognition, 2021, pp. 2398-2407.

[6] A. Ignatov, J. Patel, and R. Timofte, "Rendering natural camera bokeh effect with deep learning," in Proceedings of the IEEE/CVF Conference on Computer Vision and Pattern Recognition Workshops, 2020, pp. 418 419.

[7] D. Liu, R. Nicolescu, and R. Klette, "Bokeh effects based on stereo vision," in International Conference on Computer Analysis of Images and Patterns. Springer, 2015, pp. 198-210.

[8] C. Luo, Y. Li, K. Lin, G. Chen, S.-J. Lee, J. Choi, Y. F. Yoo, and M. O. Polley, "Wavelet synthesis net for disparity estimation to synthesize dslr calibre bokeh effect on smartphones," in Proceedings of the IEEE/CVF Conference on Computer Vision and Pattern Recognition, 2020, pp. $2407-2415$.

[9] X. Shen, A. Hertzmann, J. Jia, S. Paris, B. Price, E. Shechtman, and I. Sachs, "Automatic portrait segmentation for image stylization," in Computer Graphics Forum, vol. 35, no. 2. Wiley Online Library, 2016, pp. 93-102.
[10] N. Wadhwa, R. Garg, D. E. Jacobs, B. E. Feldman, N. Kanazawa, R. Carroll, Y. Movshovitz-Attias, J. T. Barron, Y. Pritch, and M. Levoy, "Synthetic depth-of-field with a single-camera mobile phone," ACM Transactions on Graphics (ToG), vol. 37, no. 4, pp. 1-13, 2018.

[11] S. Dutta, "Depth-aware blending of smoothed images for bokeh effect generation," Journal of Visual Communication and Image Representation, vol. 77, p. 103089, 2021

[12] Z. Li and N. Snavely, "Megadepth: Learning single-view depth prediction from internet photos," in Proceedings of the IEEE Conference on Computer Vision and Pattern Recognition, 2018, pp. 2041-2050.

[13] A. Ignatov, J. Patel, R. Timofte, B. Zheng, X. Ye, L. Huang, X. Tian, S. Dutta, K. Purohit, P. Kandula et al., "Aim 2019 challenge on bokeh effect synthesis: Methods and results," in 2019 IEEE/CVF International Conference on Computer Vision Workshop (ICCVW). IEEE, 2019, pp. 3591-3598.

[14] X. Li, W. Wang, X. Hu, and J. Yang, "Selective kernel networks," in Proceedings of the IEEE/CVF Conference on Computer Vision and Pattern Recognition, 2019, pp. 510-519.

[15] K. He, X. Zhang, S. Ren, and J. Sun, "Deep residual learning for image recognition," in Proceedings of the IEEE conference on computer vision and pattern recognition, 2016, pp. 770-778.

[16] A. Vaswani, N. Shazeer, N. Parmar, J. Uszkoreit, L. Jones, A. N. Gomez, Ł. Kaiser, and I. Polosukhin, "Attention is all you need," in Advances in neural information processing systems, 2017, pp. 5998-6008.

[17] A. Paszke, S. Gross, F. Massa, A. Lerer, J. Bradbury, G. Chanan, T. Killeen, Z. Lin, N. Gimelshein, L. Antiga et al., "Pytorch: An imperative style, high-performance deep learning library," Advances in neural information processing systems, vol. 32, pp. 8026-8037, 2019.

[18] H. Zhao, O. Gallo, I. Frosio, and J. Kautz, "Loss functions for image restoration with neural networks," IEEE Transactions on computational imaging, vol. 3, no. 1, pp. 47-57, 2016.

[19] Z. Wang, E. P. Simoncelli, and A. C. Bovik, "Multiscale structural similarity for image quality assessment," in The Thrity-Seventh Asilomar Conference on Signals, Systems \& Computers, 2003, vol. 2. Ieee, 2003, pp. 1398-1402.

[20] E. S. Paul and J. Anitha, "Chapter 5 - analysis of transform-based compression techniques for mri and ct images," in Intelligent Data Analysis for Biomedical Applications, ser. Intelligent Data-Centric Systems, D. J. Hemanth, D. Gupta, and V. Emilia Balas, Eds. Academic Press, 2019, pp. 103-120.

[21] R. Zhang, P. Isola, A. A. Efros, E. Shechtman, and O. Wang, "The unreasonable effectiveness of deep features as a perceptual metric," in Proceedings of the IEEE conference on computer vision and pattern recognition, 2018, pp. 586-595.

[22] D. P. Kingma and J. Ba, "Adam: A method for stochastic optimization," arXiv preprint arXiv:1412.6980, 2014. 